\title{
On the origin of oscillatory interactions between surfaces mediated by polyelectrolyte solution
}

Cite as: J. Chem. Phys. 151, 214901 (2019); https://doi.org/10.1063/1.5123172

Submitted: 05 August 2019. Accepted: 13 November 2019. Published Online: 02 December 2019

Jian Jiang, Valeriy V. Ginzburg, and Zhen-Gang Wang (D)

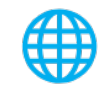




\title{
On the origin of oscillatory interactions between surfaces mediated by polyelectrolyte solution
}

\author{
Cite as: J. Chem. Phys. 151, 214901 (2019); doi: 10.1063/1.5123172 \\ Submitted: 5 August 2019 - Accepted: 13 November 2019• \\ Published Online: 2 December 2019
}

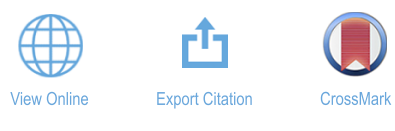

Jian Jiang, ${ }^{1,2,3}$ Valeriy V. Ginzburg, ${ }^{4}$ and Zhen-Gang Wang

\begin{abstract}
AFFILIATIONS
${ }^{1}$ Division of Chemistry and Chemical Engineering, California Institute of Technology, Pasadena, California 91125, USA

${ }^{2}$ Beijing National Laboratory for Molecular Sciences, State Key Laboratory of Polymer Physics and Chemistry, Institute of Chemistry, Chinese Academy of Sciences, Beijing 100190, People's Republic of China

${ }^{3}$ University of Chinese Academy of Sciences, Beijing 100049, People's Republic of China

${ }^{4}$ Research and Development, The Dow Chemical Company, Midland, Michigan 48674, USA
\end{abstract}

a) Author to whom correspondence should be addressed: zgw@caltech.edu

\begin{abstract}
We use a numerical implementation of polymer classical density functional theory with an incompressibility condition to study the system consisting of nonadsorbing polyelectrolytes confined by two planar surfaces and quantify the effective interaction between the two planar surfaces as a function of the polyelectrolyte and salt concentrations. Our results indicate that for the uncharged surfaces (and weakly charged surfaces), the effective interaction primarily consists of a short-range attraction due to the depletion followed by repulsion due to the electric double layer overlapping and electrostatic correlations. For salt-free and low salt concentration systems, the magnitude of the repulsion barrier is determined by the overlap between the electric double layers, while at relatively high salt concentrations, the magnitude of the repulsion barrier is determined by the electrostatic correlations. Due to the competition between the electric double layer and the electrostatic correlations, the magnitude of the repulsion barrier varies nonmonotonically. In contrast, a mean-field Poisson-Boltzmann treatment of the electrostatics predicts a monotonically decreasing repulsion barrier with increasing salt concentration. At moderate salt concentrations, our theory predicts oscillatory interaction profiles. A comparison with the mean-field Poisson-Boltzmann treatment of electrostatics suggests that the oscillations are due primarily to electrostatic correlations.
\end{abstract}

Published under license by AIP Publishing. https://doi.org/10.1063/1.5123172

\section{INTRODUCTION}

Because of the complex interplay between chain connectivity, excluded volume interaction, and long-ranged Coulomb interactions, the effective interaction between surfaces mediated by polyelectrolyte solutions can exhibit unusual behaviors. ${ }^{1-6}$ For this reason, polyelectrolytes are extensively used in industries as additives in various applications, such as flocculation and stabilization of colloidal suspensions, emulsions and foams, water purification, and papermaking. ${ }^{7-9}$ A particularly interesting property is the oscillation in the force between two surfaces (solid/aqueous interfaces and liquid films) mediated by polyelectrolyte solutions as a function of the separation; ${ }^{4,5,10-13}$ the dependence on polyelectrolyte and salt concentrations makes it possible to modulate the force by changing these variables.
Simple fluids at liquid densities confined between two surfaces are known to induce oscillatory forces as a function of surface separation. ${ }^{14-17}$ The oscillations in this case are attributed to the layering of the liquid in the confining surfaces due to packing at the liquid densities; the oscillations disappear at reduced densities. The origin of the oscillatory forces in polyelectrolyte solutions is less obvious, although the oscillatory-force behavior induced by polyelectrolytes has been extensively investigated during the last two decades.

In contrast to simple liquids, the oscillating interactions between confined polyelectrolyte solutions are caused not by packing at the monomer length scales, but by the structures at mesoscopic scales. Dilute or semidilute solutions are known to form bulk structures (such as networks, stratification, liquidlike packing, and cylinderlike packing), which have been measured using small-angle scattering method (SAXS or SANS) ${ }^{25}$ and other techniques. 
These bulk structures have been invoked to interpret the oscillatory forces of confined polyelectrolytes solutions. $5,11,13,19,21,22,26-29$ However, bulk structures such as networks exist not only in the polyelectrolytes solutions but also in the uncharged polymer solutions. Yet, oscillatory forces are not observed in confined but ungrafted uncharged polymer systems. In addition, contradicting results have been reported in the literature regarding the nature of effective interactions between neutral or weekly charged surfaces: For example, some theoretical works and computer simulations $^{11,21}$ show short-range repulsions, while other theoretical works $^{30}$ and computer simulations ${ }^{26}$ show short-range attractions. Also, some theoretical works ${ }^{21}$ and computer simulations ${ }^{31}$ predict that the amplitude of the oscillations decreases with increasing polymer concentration, whereas experiments ${ }^{19}$ show the opposite. Despite the contradictory results and differing explanations, it is generally agreed that, in the semidilute regime where most of the experiments and simulations were performed, the length scale of the interaction force is governed by the correlation length of the solution, which is a function of the concentration but not of the chain length. ${ }^{4,5,13,21-24,32}$ For example, Ref. 24 showed convincingly that the range of the attractive interaction in the interaction profile between two nonionic surfactant layers in a free-standing salt-free polyelectrolyte solution matched quantitatively with the correlation length of the solution as measured from small-angle neutron scattering.

In this work, we use a polymer classical density functional theory (PDFT) based on our previous work ${ }^{33}$ to study the interactions between two neutral hard walls induced by the polyelectrolytes/salt solutions. Our results show that the primary feature of the interaction profile is a short-range attraction followed by repulsion at larger distance. At moderate salt concentrations, we find oscillatory interaction profile, with the amplitude increasing with polymer concentration, in agreement with experiments. ${ }^{19}$ By comparison with the mean-field Poisson-Boltzmann (PB) treatment of electrostatics, we conclude that the oscillatory forces are primarily due to electrostatic correlations.

The rest of this article is organized as follows: In Sec. II, we present the model and the PDFT formulated with the incompressibility condition, and we briefly explain the numerical implementation of our theory in Sec. III. In Sec. IV, we find that for salt-free and low-salt conditions, the interaction is controlled by the overlap between the electrical double layers (EDL) from each surface, while at high salt concentrations, electrostatic correlations play a key role. The competition between these two effects results in oscillatory interactions at moderate salt concentrations. In Sec. V, we conclude with a brief summary and outlook.

\section{MOLECULAR MODEL AND THEORY}

The polyelectrolyte solution is characterized by the polyion concentration $\rho_{\mathrm{p}}$ (where the subscript $\mathrm{p}$ refers to the polymer monomers), polyion chain length $N$, salt concentration $\rho_{ \pm}$, and ionic valences $Z_{i^{\prime}}$ (where $i^{\prime}=\mathrm{p},+,-$ is the index of the species). The polyelectrolyte chains are represented by tangentially connected charged hard spheres, which was first introduced into the PDFT framework by Woodward, ${ }^{34}$ with diameter $\sigma_{\mathrm{p}}$; the couterions and salts are modeled as charged hard spheres as well; and the solvent is treated as hard spheres with diameter $\sigma_{\mathrm{s}}$ with a continuum dielectric background. For concreteness, we assume each monomer on the polyion chain carries one negative charge (see Fig. 1). We further assume the counterions and salt cations are identical.

Commonly, for solutions, there are three different treatments of the solvent in the framework of PDFT: (1) explicitly treating the solvent as an additional component; (2) neglecting the solvent (implicit solvent); and (3) accounting for the solvent using incompressibility. Liquid-state based PDFT typically assumes implicit solvent. ${ }^{35,36}$ The second is the most commonly used. The third is commonly used as the continuum self-consistent field treatment of polymers. ${ }^{37}$ In the case of very dilute solutions, the second and the third treatments are equivalent except when the surfaces are strongly attractive. Forsman and co-workers have shown that in the neutral polymer systems, different treatments of the solvent predict different results, and they observed that when the solvent is considered explicitly, the solvent has important effects on the interaction behavior between the surfaces.

Both the explicit and implicit treatments introduce strong local packing effects that may not be physical: In the explicit-solvent treatment, the hard-sphere nature of the solvent results lead to solidlike packing near the wall, while in the implicit-solvent model, there is an intrinsic asymmetry between the packing of the solvent and the solutes. As these features are concerned with the structures at the monomer length scales, in order to focus on the larger scale properties, we adopt the third treatment of the solvent in PDFT with the incompressibility condition. To the usual PDFT, we add the incompressibility condition: $\sum_{i} \rho_{i}(\mathbf{r}) v_{i}=\eta$, where $v_{i}=\pi \sigma_{i}^{3} / 6$ is the volume of the species $i$ (where $i=\mathrm{p},+,-, \mathrm{s}$ ) and $\eta$ is a constant during the calculations, which we choose to be 0.5 . In essence, this condition is a localized version of the statement of no volume change in mixing the different species, an assumption that is reasonable for the liquid state. Note that this condition is imposed upon a free energy functional that uses an explicit solvent model, which by itself would produce packing-induced variations in the volume fraction or density. The local incompressibility condition smears out such variations and therefore amounts to some kind of spatial coarse-graining (averaging). Because of the incompressibility condition, the total hydrostatic pressure is no longer meaningful; however, the osmotic pressure of the species can still be meaningfully calculated.

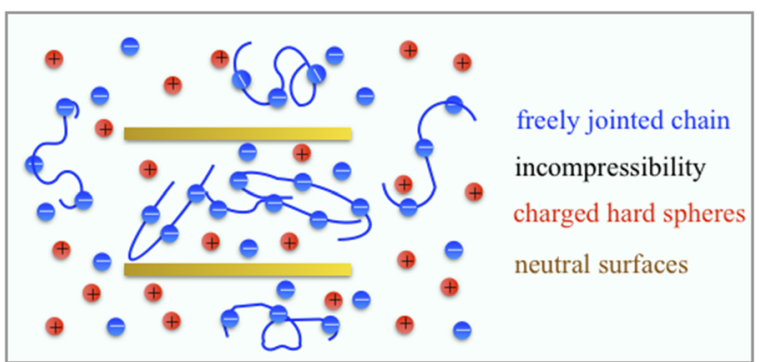

FIG. 1. Schematic of the model under consideration in this work. We consider system consisting of two neutral planar surfaces immersed in a solution of negatively charged polyelectrolytes and salts with uniform dielectric constant $\epsilon_{\mathrm{r}}=80$. The temperature is $300 \mathrm{~K}$, and the diameter of the monomers, counterions, salts, and solvents is $5 \AA$ 
PDFT is based on the idea that the Helmholtz free energy, or equivalently, the grand potential, can be fully specified as a functional of the density distributions. For polyelectrolytes systems with incompressibility condition considered in this work, the grand potential can be written as

$$
\begin{aligned}
& W\left[\rho_{\mathrm{p}}(\mathbf{R}),\left\{\rho_{\alpha}(\mathbf{r})\right\}, \rho_{\mathrm{s}}(\mathbf{r}), \phi(\mathbf{r})\right] \\
& =F_{\mathrm{id}}+\int \mathrm{d} \mathbf{r} f_{\mathrm{ex}}\left[\left\{\rho_{i}(\mathbf{r})\right\} ; \mathbf{r}\right]+\int \mathrm{d} \mathbf{R} \rho_{\mathrm{p}}(\mathbf{R})\left[\Psi_{\mathrm{p}}(\mathbf{R})-\mu_{\mathrm{p}}\right] \\
& +\sum_{\alpha} \int \mathrm{d} \mathbf{r} \rho_{\alpha}(\mathbf{r})\left[\psi_{\alpha}(\mathbf{r})-\mu_{\alpha}\right]+\int \mathrm{d} \mathbf{r} \rho_{\mathrm{s}}(\mathbf{r})\left[\psi_{\mathrm{s}}(\mathbf{r})-\mu_{\mathrm{s}}\right] \\
& \quad+\int \mathrm{d} \mathbf{r} \lambda(\mathbf{r})\left[\sum_{i} v_{i} \rho_{i}(\mathbf{r})-\eta\right]
\end{aligned}
$$

where $\mathbf{R}$ represents the $3 N$-dimensional position vector for the entire chain and $d \mathbf{R}=\mathrm{dr}_{1}, \mathrm{dr}_{2}, \ldots, \mathrm{dr}_{N}$. Note that $\rho_{\mathrm{p}}(\mathrm{R})$ is the $3 N$-dimensional density distribution of the polymer chains, whereas $\rho_{\mathrm{p}}(\mathrm{r})$ is the 3-dimensional density distribution of the total monomers. In this work, $i$ refers to solvent(s), small ions $( \pm)$, and polyelectrolytes (p); $i^{\prime}$ means small ions and polyelectrolytes; $\alpha$ refers to small ions (salts and counterions) only. $\Psi_{\mathrm{p}}(\mathrm{R})$ is the external potential that acts on individual segments of polymers, i.e., $\Psi_{\mathrm{p}}(\mathbf{R})$ $=\sum_{m=1}^{N} \psi_{\mathrm{p}}\left(\mathbf{r}_{m}\right) ; \psi_{\alpha}(\mathbf{r})$ and $\psi_{s}(\mathbf{r})$ are the external potential for small ions and solvent, respectively; and $\mu_{\mathrm{s}}, \mu_{\alpha}$, and $\mu_{\mathrm{p}}$ are, respectively, the chemical potentials of solvent, small ions, and polyelectrolytes. $\lambda(\mathbf{r})$ is Lagrange's multiplier. The overall segment density $\rho_{\mathrm{p}}(\mathbf{r})$ is related to $\rho_{\mathrm{p}}(\mathbf{R})$ via

$$
\rho_{\mathrm{p}}(\mathbf{r})=\sum_{m=1}^{N} \rho_{\mathrm{s} m}(\mathbf{r})=\sum_{m=1}^{N} \int \mathrm{d} \mathbf{R} \delta\left(\mathbf{r}-\mathbf{r}_{m}\right) \rho_{\mathrm{p}}(\mathbf{R}),
$$

where $\delta(\mathbf{r})$ is the Dirac-delta function.

In Eq. (2.1), the ideal free energy is given by

$$
\begin{aligned}
\beta F_{\mathrm{id}}= & \int \mathrm{d} \mathbf{R} \rho_{\mathrm{p}}(\mathbf{R})\left\{\ln \left[\rho_{\mathrm{p}}(\mathbf{R}) a_{\mathrm{p}}^{N}\right]-1\right\}+\int \mathrm{d} \mathbf{R} \rho_{\mathrm{p}}(\mathbf{R}) \beta V_{\mathrm{b}}(\mathbf{R}) \\
& +\sum_{\alpha} \int \mathrm{d} \mathbf{r} \rho_{\alpha}(\mathbf{r})\left\{\ln \left[\rho_{\alpha}(\mathbf{r}) a_{\alpha}\right]-1\right\} \\
& +\int \mathrm{d} \mathbf{r} \rho_{\mathrm{s}}(\mathbf{r})\left\{\ln \left[\rho_{\mathrm{s}}(\mathbf{r}) a_{\mathrm{s}}\right]-1\right\},
\end{aligned}
$$

where $a_{i}$ is a volume scale that can be taken as the cube of the thermal de Broglie length; the precise definition of $a_{i}$ is immaterial as it has no thermodynamic consequences. The bonding potential $V_{\mathrm{b}}(\mathbf{R})$ in the ideal part [Eq. (2.3)] is written in the form

$$
\exp \left[-\beta V_{\mathrm{b}}(\mathbf{R})\right]=a_{\mathrm{p}}^{N-1} \prod_{m=1}^{N-1} \frac{\delta\left(\left|\mathbf{r}_{m+1}-\mathbf{r}_{m}\right|-\sigma_{\mathrm{p}}\right)}{4 \pi \sigma_{\mathrm{p}}^{2}} .
$$

Equation (2.4) is for the freely jointed chain model. It can be modified to account for bending stiffness as shown by Forsman and Woodward. ${ }^{39,40}$ The excess free energy density $f_{\text {ex }}$ in Eq. (2.1) is the sum of $f_{\mathrm{hs}}, f_{\mathrm{C}}, f_{\mathrm{el}}, f_{\mathrm{ch}}$, and $f_{\mathrm{vdW}}$, which account, respectively, for the hard-core excluded volume interaction, the (bare) Coulomb interaction, nonbonded electrostatic correlation, chain connectivity correlation, and van der Waals attraction. $f_{\mathrm{vdW}}$ is usually treated using a mean-field approximation. ${ }^{41}$ In order to highlight the effects of electrostatic interactions on the force behavior between two surfaces, we ignore this part of the interaction in this work. The Coulomb interaction $f_{\mathrm{C}}$ is given by

$$
f_{\mathrm{C}}\left[\phi(\mathbf{r}),\left\{\rho_{i^{\prime}}(\mathbf{r})\right\} ; \mathbf{r}\right]=\sum_{i^{\prime}} \phi(\mathbf{r}) Z_{i^{\prime}} e_{0} \rho_{i^{\prime}}(\mathbf{r})-\frac{\epsilon \epsilon_{0}}{2}[\nabla \phi(\mathbf{r})]^{2},
$$

where $\phi(\mathbf{r})$ is the electrostatic potential.

For the nonbonded electrostatic correlation, we perform the functional Taylor expansion by writing

$$
\begin{aligned}
f_{\mathrm{el}}\left[\left\{\rho_{i^{\prime}}(\mathbf{r})\right\} ; \mathbf{r}\right] \approx & f_{\mathrm{el}}\left[\left\{\rho_{i^{\prime}}^{\mathrm{ref}}(\mathbf{r})\right\} ; \mathbf{r}\right]+\sum_{i^{\prime}} \int \mathrm{d} \mathbf{r} \frac{\delta f_{\mathrm{el}}\left[\left\{\rho_{i^{\prime}}^{\mathrm{ref}}(\mathbf{r})\right\}\right]}{\delta \rho_{i^{\prime}}^{\mathrm{ref}}(\mathbf{r})} \\
& \times\left[\rho_{i^{\prime}}(\mathbf{r})-\rho_{i^{\prime}}^{\mathrm{ref}}(\mathbf{r})\right]+\cdots,
\end{aligned}
$$

where the nonbonded electrostatic correlation for the reference fluids is obtained using mean spherical approximation (MSA), ${ }^{42-45}$ with the result

$$
\beta f_{\mathrm{el}}\left[\left\{\rho_{i^{\prime}}^{\mathrm{ref}}(\mathbf{r})\right\} ; \mathbf{r}\right]=\frac{\Gamma^{3}(\mathbf{r})}{3 \pi}-l_{\mathrm{B}} \sum_{i} \rho_{i} Z_{i}\left(\frac{Z_{i}-Z_{i}^{\mathrm{eff}}}{\sigma_{i}}\right),
$$

where $\Gamma(\mathbf{r})$ is the MSA screening parameter (with units of inverse length), and $l_{\mathrm{B}}=\beta e_{0}^{2} / 4 \pi \epsilon \epsilon_{0}$ is the Bjerrum length with $e_{0}$ and $\epsilon \epsilon_{0}$ being the elementary charge and electric permittivity, respectively. The screening parameter $\Gamma$ is obtained from the implicit relation

$$
\Gamma^{2}(\mathbf{r})=\pi l_{\mathrm{B}} \sum_{i^{\prime}} \rho_{i^{\prime}}^{\mathrm{ref}}(\mathbf{r})\left(Z_{i^{\prime}}^{\mathrm{eff}}\right)^{2}
$$

where $Z_{i^{\prime}}^{\text {eff }}=\left(Z_{i^{\prime}}-\chi \sigma_{i^{\prime}}{ }^{2}\right) /\left(1+\Gamma \sigma_{i^{\prime}}\right)$ can be considered as the effective valency, and $\chi$ is determined by

$$
\chi=\sum_{i^{\prime}} \frac{\rho_{i^{\prime}}^{\mathrm{ref}}(\mathbf{r}) \sigma_{i^{\prime}} Z_{i^{\prime}}}{1+\Gamma \sigma_{i^{\prime}}} /\left[\frac{2(1-\eta)}{\pi}+\sum_{i^{\prime}} \frac{\rho_{i^{\prime}}^{\mathrm{ref}}(\mathbf{r}) \sigma_{i^{\prime}}^{3}}{1+\Gamma \sigma_{i^{\prime}}}\right] .
$$

For point charges (i.e., when the ion diameters are zero) in a uniform bulk, $Z_{i^{\prime}}^{\text {eff }}=Z_{i^{\prime}}$, and $1 /(2 \Gamma)$ is simply the Debye screening length. The simplest choice of the reference fluids $\left\{\rho_{i}^{\text {ref }}(\mathbf{r})\right\}$ is to take the uniform bulk densities. ${ }^{46}$ However, as shown in Refs. 47 and 48, this scheme can result in qualitative discrepancies with simulation results for asymmetric ions near weakly charged surfaces. In this work, we adopt the scheme proposed by Gillespie and co-workers, ${ }^{49}$ termed the reference fluid density (RFD) and only keep the linear term (the first order) in the RHS of Eq. (2.6). We have discussed in Ref. 33 that the first-order approximation gives nearly the same results with those from the second-order approximation. A brief recapitulation of the key ideas and implementation of RFD is presented in the Appendix of Ref. 33.

The first-order TPT (TPT-1) ${ }^{50-54}$ has been successfully employed to describe the structure and thermodynamic properties of inhomogeneous uncharged polymer systems. ${ }^{55-59}$ For polyelectrolyte solutions, as discussed in Ref. 33, the connectivity correlation can be written as

$$
f_{\mathrm{ch}}\left[\left\{\rho_{i^{\prime}}(\mathbf{r})\right\} ; \mathbf{r}\right]=f_{\mathrm{ch}}^{\mathrm{hs}}\left[\left\{\rho_{i^{\prime}}(\mathbf{r})\right\} ; \mathbf{r}\right]+f_{\mathrm{ch}}^{\mathrm{el}}\left[\left\{\rho_{i^{\prime}}(\mathbf{r})\right\} ; \mathbf{r}\right],
$$

where the first term in the RHS of Eq. (2.10) is the hard-sphere contribution, and the second term is the contribution from electrostatic correlation. We have

$$
f_{\mathrm{ch}}^{\mathrm{hs}}\left[\left\{\rho_{i^{\prime}}(\mathbf{r})\right\} ; \mathbf{r}\right]=\frac{(1-N) \rho_{\mathrm{p}}(\mathbf{r})}{N} \ln g\left(\sigma_{\mathrm{p}},\left\{\rho_{i^{\prime}}(\mathbf{r})\right\}\right)
$$


and

$$
\begin{aligned}
f_{\mathrm{ch}}^{\mathrm{el}}\left[\left\{\rho_{i^{\prime}}(\mathbf{r})\right\} ; \mathbf{r}\right] \approx & f_{\mathrm{ch}}^{\mathrm{el}}\left[\left\{\rho_{i^{\prime}}^{\mathrm{ref}}(\mathbf{r})\right\} ; \mathbf{r}\right]+\sum_{i^{\prime}} \int \mathrm{d} \mathbf{r} \frac{\delta f_{\mathrm{ch}}^{\mathrm{el}}\left[\left\{\rho_{i^{\prime}}^{\mathrm{ref}}(\mathbf{r})\right\}\right]}{\delta \rho_{i^{\prime}}^{\mathrm{ref}}(\mathbf{r})} \\
& \times\left[\rho_{i^{\prime}}(\mathbf{r})-\rho_{i^{\prime}}^{\mathrm{ref}}(\mathbf{r})\right]+\cdots,
\end{aligned}
$$

where the electrostatic correlation part for reference fluids in Eq. (2.12) is

$$
f_{\mathrm{ch}}^{\mathrm{el}}\left[\left\{\rho_{i^{\prime}}^{\mathrm{ref}}(\mathbf{r})\right\} ; \mathbf{r}\right]=\frac{(1-N) l_{\mathrm{B}} \rho_{\mathrm{p}}^{\mathrm{ref}}(\mathbf{r})\left[Z_{i}^{2}-\left(Z_{i}^{\mathrm{eff}}\right)^{2}\right]}{N \sigma_{\mathrm{p}}} .
$$

To maintain the same level of accuracy with the nonbonded electrostatic correlation in Eq. (2.6), we only retain the linear term in the RHS of Eq. (2.12). In Eq. (2.11), the $g\left(\sigma_{\mathrm{p}},\left\{\rho_{i^{\prime}}(\mathbf{r})\right\}\right)$ is the pair distribution function at contact for given densities $\left\{\rho_{i^{\prime}}(\mathbf{r})\right\}^{3}$

As in any DFTs including self-consistent field theories for polymers, our DFT does not accurately capture the conformation properties of the polymers in dilute and semidilute solutions due to the use of the ideal chain model in describing the chain statistics [Eq. (2.4)] and the TPT-1 treatment of the correlations due to chain connectivity [Eq. (2.10)]. These approximations are expected to lead to underestimate of the correlation effects, including the correlation length. For example, the correlation length of salt-free polyelectrolyte solutions in the semidilute regime should scale as $\xi \sim \rho_{\mathrm{p}}^{-1 / 2}$, where the prefactor reflects the local rigidity of the blobs due to electrostatic repulsion of the chain segments. ${ }^{60}$ By assuming ideal chain statistics, our DFT greatly underestimates the value of the prefactor. Therefore, in our numerical calculations, we choose to work in the concentrated solutions where the chain conformation is approximately ideal. Nevertheless, we expect many of the qualitative features to extend to the lower concentration regimes. We note that for bulk solutions, there exist theories that correctly describe the chain conformation by allowing it to adapt to the polymer and salt concentrations, such as the "double screening" theory ${ }^{61}$ and the renormalized Gaussian fluctuation theory; ${ }^{62}$ however, extension of these theories to inhomogeneous systems is difficult.

There are three common approaches of DFT to the inhomogeneous excess free energy functional due to the hard-sphere excluded volume effect, i.e., $f_{\mathrm{hs}}$. One approach is the weighted density approximation (WDA) ${ }^{63-70}$ combined with a suitable equation of state (EOS). The other approach is based on the fundamental measure theory (FMT) $)^{71,72}$ and its modifications (MFMT) ${ }^{73,74}$ The simplest scheme for describing the inhomogeneous excess free energy functional due to the hard-spheres is the local density approximation (LDA). The LDA approximates the local excess free energy density for hard sphere contribution as a function of the local densities. The description of the hard-sphere contribution from LDA is reasonable if we are interested in longer-length scale structures than those for the packing of the monomers and solvent molecules. ${ }^{75,76}$ In this work, we concentrate on studying the electrostatic correlations and chain connectivities. To this end, we combine the LDA with the Boublík-Mansoori-Carnarhan-Starling-Leland (BMCSL) $\operatorname{EOS}^{77,78}$ to approximate the excess free energy density due to the hard-sphere contributions given by

$$
\begin{aligned}
\beta f_{\mathrm{hs}}\left[\left\{\rho_{i}(\mathbf{r})\right\} ; \mathbf{r}\right]= & -\xi_{0} \ln \left(1-\xi_{3}\right)+\frac{\xi_{1} \xi_{2}}{\left(1-\xi_{3}\right)}+\frac{\xi_{2}^{3}}{36 \pi} \\
& \times\left[\frac{\ln \left(1-\xi_{3}\right)}{\xi_{3}^{2}}+\frac{1}{\xi_{3}\left(1-\xi_{3}\right)^{2}}\right],
\end{aligned}
$$

where $\xi_{3}=\pi \sum_{i} \rho_{i}(\mathbf{r}) \sigma_{i}^{3} / 6, \xi_{2}=\pi \sum_{i} \rho_{i}(\mathbf{r}) \sigma_{i}^{2}, \xi_{1}=\sum_{i} \rho_{i}(\mathrm{r}) \sigma_{i} / 2$, and $\xi_{0}=\sum_{i} \rho_{i}(\mathrm{r})$. Note that $\xi_{3}$ is the local total packing fraction. If the sizes of the monomers, counterions, and salts are the same with the size of solvent, Eq. (2.14) will not contribute to the Euler-Lagrange equations as shown in Eqs. (2.15)-(2.17) due to the zero exchange chemical potential (i.e., $\partial f_{\mathrm{hs}} / \partial \rho_{i^{\prime}}=0$ ) in the incompressible systems. Although in this work we only consider the case where all the components have the same monomer sizes, we retain the term $f_{\mathrm{hs}}$ in order to present a general theory that can be used to study cases with arbitrary sizes for the species.

Extremization of the grand potential [Eq. (2.1)] with respect to $\rho_{\mathrm{p}}(\mathbf{R}), \rho_{\alpha}(\mathbf{r}), \rho_{\mathrm{s}}(\mathbf{r}), \lambda(\mathbf{r})$, and $\phi(\mathbf{r})$ yields, respectively, the EulerLagrange equations for the density profiles and the Lagrange's multiplier, and the Poisson equation,

$$
\begin{gathered}
\rho_{\mathrm{p}}(\mathbf{R}) a_{\mathrm{p}}^{N}=\exp \left\{\beta\left[\mu_{\mathrm{p}}-V_{\mathrm{b}}(\mathbf{R})-\sum_{m=1}^{N} \Lambda_{\mathrm{p}}\left(\mathbf{r}_{m}\right)\right]\right\}, \\
\rho_{\alpha}(\mathbf{r}) a_{\alpha}=\exp \left\{\beta\left[\mu_{\alpha}-\Lambda_{\alpha}\left(\mathbf{r}_{m}\right)\right]\right\}, \\
\beta \lambda v_{s}=\beta\left[\mu_{s}-\psi_{s}(\mathbf{r})-\frac{\partial f_{\mathrm{ex}}}{\partial \rho_{s}(\mathbf{r})}\right]-\ln \rho_{s}(\mathbf{r}) a_{s},
\end{gathered}
$$

and

$$
\nabla^{2} \phi(\mathbf{r})=-\frac{1}{\epsilon \epsilon_{0}} \sum_{i^{\prime}} Z_{i} e_{0} \rho_{i}(\mathbf{r})
$$

with the incompressibility constraint $\sum_{i} v_{i} \rho_{i}(\mathrm{r})-\eta=0$. In Eqs. (2.15) and (2.16), the effective fields $\Lambda_{\mathrm{p}}(\mathrm{r})$ and $\Lambda_{\alpha}(\mathrm{r})$ are

$$
\Lambda_{\mathrm{p}}(\mathbf{r})=\frac{\partial f_{\mathrm{ex}}}{\partial \rho_{\mathrm{p}}(\mathbf{r})}+\psi_{\mathrm{p}}(\mathbf{r})+Z_{\mathrm{p}} e_{0} \phi(\mathbf{r})+\lambda v_{\mathrm{p}}
$$

and

$$
\Lambda_{\alpha}(\mathbf{r})=\frac{\partial f_{\mathrm{ex}}}{\partial \rho_{\alpha}(\mathbf{r})}+\psi_{\alpha}(\mathbf{r})+Z_{\alpha} e_{0} \phi(\mathbf{r})+\lambda v_{\alpha} .
$$

\section{NUMERICAL IMPLEMENTATION}

For the slit pore geometry considered in this work, the density profiles of monomers and small ions vary only in the $z$ direction, and we have

$$
\rho_{\mathrm{p}}(z) a_{\mathrm{p}}=\exp \left(\beta \mu_{\mathrm{p}}\right) \sum_{m=1}^{N} \exp \left[-\beta \Lambda_{\mathrm{p}}(z)\right] G_{\mathrm{L}}^{m}(z) G_{\mathrm{R}}^{m}(z)
$$

and

$$
\rho_{\alpha}(z) a_{\alpha}=\exp \left\{\left[\beta \mu_{\alpha}-\beta \Lambda_{\alpha}(z)\right]\right\} .
$$

The left propagator $G_{\mathrm{L}}^{m}(z)$ in Eq. (3.1) is determined from the recurrence relation

$$
G_{\mathrm{L}}^{m}(z)=\frac{1}{2 \sigma_{\mathrm{p}}} \int_{z-\sigma_{\mathrm{p}}}^{z+\sigma_{\mathrm{p}}} \mathrm{d} z^{\prime} \exp \left[-\beta \lambda_{\mathrm{p}}\left(z^{\prime}\right)\right] G_{\mathrm{L}}^{m-1}\left(z^{\prime}\right),
$$


for $m=2, \ldots, N$ with $G_{\mathrm{L}}^{1}(z)=1$. For homopolymers, the right propagator is related to the left propagator via $G_{\mathrm{R}}^{m}(z)=G_{\mathrm{L}}^{N+1-m}(z)$.

The mean electrostatic potential $\phi(z)$ is given by

$$
\begin{aligned}
\beta e_{0} \phi(z)= & \beta e_{0} \phi(0)+4 \pi l_{\mathrm{B}} \sum_{i} \int_{0}^{h} Z_{i} \rho_{i}\left(z^{\prime}\right)\left(z-z z^{\prime} / h\right) \mathrm{d} z^{\prime} \\
& +4 \pi l_{\mathrm{B}} \sum_{i} \int_{0}^{z} Z_{i} \rho_{i}\left(z^{\prime}\right)\left(z^{\prime}-z\right) \mathrm{d} z^{\prime} .
\end{aligned}
$$

The potential at the surface $\phi(0)$ is determined from the boundary conditions $\mathrm{d} \phi(z) /\left.\mathrm{d} z\right|_{z=0}=-\mathrm{d} \phi(z) /\left.\mathrm{d} z\right|_{z=h}=-Q / \epsilon \epsilon_{0}$ and charge neutrality

$$
\sum_{i} \int_{0^{+}}^{h^{-}} Z_{i} e_{0} \rho_{i}\left(z^{\prime}\right) \mathrm{d} z^{\prime}+2 Q=0,
$$

where $Q$ is the surface charge density and $h$ is the width of the slit pore. In this work, we only consider neutral hard-wall surfaces $(Q=0)$ in order to focus on studying the effects of induced electric double layers and electrostatic correlations on the force behaviors. Electrostatic and nonelectrostatic adsorption effects will be addressed in future work.

Equations (3.1)-(3.4) are solved by Picard iteration. The iteration starts with the bulk densities of polyelectrolytes and small ions as input, denoted by $\left\{\rho_{i}^{\text {old }}(z)\right\}$. Then, the effective fields $\left\{\lambda_{i}(z)\right\}$ are calculated, which result in the new density profiles $\left\{\rho_{i}^{*}(z)\right\}$ for polyelectrolytes and small ions. Then, the new input densities $\left\{\rho_{i}^{\text {new }}(z)\right\}$ are assigned by the following mixing rule: $\rho_{i}^{\text {new }}(z)=\rho_{i}^{*}(z) p_{0}$ $+\rho_{i}^{\text {old }}(z)\left(1-p_{0}\right)$, where $p_{0}$ is a mixing parameter, typically on the order of 0.01 . The procedure is repeated until the difference between successive iterations in the densities of $\left\{\rho_{i}^{\text {old }}(z)\right\}$ and $\left\{\rho_{i}^{*}(z)\right\}$ at all positions normalized by their respective bulk densities is less than $10^{-6}$.

\section{RESULTS AND DISCUSSION}

The properties of polyelectrolyte solutions are influenced by a large parameter space. Since salt concentration is a key control variable, we present our results separately for the salt-free systems and for systems with salt.

\section{A. Salt-free systems}

As a point of reference, we start by briefly reviewing the interaction force between hard surfaces in neutral polymer solution. We calculate the force per unit area between the two surfaces by $\Pi(h)$ $-\Pi(\infty)$, where $\Pi(h)=-\partial W / \partial h$. At fixed monomer concentration corresponding to low volume fraction, the polymer solution is in the semidilute regime for sufficiently long chain lengths. In this regime, depletion of the polymer near the surface induces attraction between the surfaces. The range of the depletion attraction is set by the correlation length, whereas the depth is given by the bulk osmotic pressure; ${ }^{79}$ both being independent of chain lengths for sufficiently long chains. As the chain length decreases, the overlap concentration increases, and at fixed monomer concentration, the solution becomes comparatively more dilute. In this regime, the depletion force is essentially that given by Asakura and Oosawa for isolated chains. ${ }^{80}$ Since the chain density increases, the bulk osmotic pressure increases with decreasing chain length at fixed monomer concentration, and so, the depth of the depletion attraction increases. In
Fig. 2(a), we show the interaction force profiles at a monomer concentration of $0.5 \mathrm{M}$ for several different chain lengths ranging from 10 to 100 . The observed trend is consistent with the above discussion. Although the change in the profile is more gradual for the longer chains, there is significant chain length dependence for all the four chain lengths studied.

In contrast, the polyelectrolyte-mediated force profiles shown in Fig. 2(b) are remarkably insensitive to chain lengths for the same set of chain lengths as in the neutral polymer case. This insensitivity reflects the dominant role played by the counterions at this polyelectrolyte concentration. Because each polyelectrolyte carries $N$ counterions, in the concentration regime we consider, the total ion concentration is sufficiently high that the correlation length is primarily set by the Debye screening length, which depends on the total ion concentration and is insensitive to the chain length. This correlation length sets the range of the attractive region and the position of the peak in our work. The presence of the small ions also explains the lack of the depletion plateau for neutral polymers. As long as the slit separation exceeds the ion size, the small counterions will be able to enter the pore space driven by their translational entropy. Because of charge neutrality, polyelectrolytes are dragged along by the small counterions, at the expense of some conformation entropy. Our theoretical results are consistent with both experimental result ${ }^{20}$ and simulation data, ${ }^{11}$ both of which showed only weak dependence on
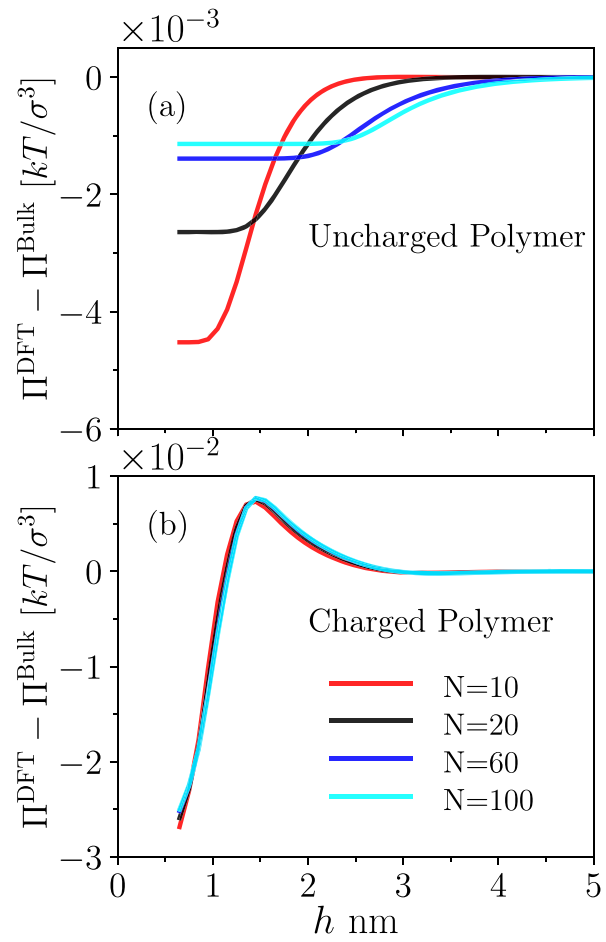

FIG. 2. Uncharged polymer/polyelectrolyte-mediated interactions between two neutral hard wall surfaces corresponding to different chain lengths. The dielectric constant of the system is uniform and set to $\epsilon=80$; the temperature is $300 \mathrm{~K}$; the monomer concentration is $0.5 \mathrm{M}$, which is comparable to the concentrations used in some experiments ${ }^{24}$ and simulations, ${ }^{11,21,26,28}$ and the diameter of the monomers, counterions, and solvent is $\sigma=5 \AA$. 
chain lengths except for a very short chain $(N<20)$. Since the profile for $N=60$ is nearly identical to that for $N=100$, in most of our discussions, we use chain length $N=60$.

The most notable feature of the normal force per unit area profiles (hereafter simply referred to as the "force" for brevity) shown in Fig. 2 is the strong attraction at shorter distances, followed by a repulsive peak at larger distances. This general feature is preserved as we change the polyelectrolyte concentration. Figure 3 shows the force profiles for several values of the polyelectrolyte concentration. A close inspection of the force profile shows a secondary attractive well to the right of the repulsion peak. Thus, the interaction profile is oscillatory. Clearly, the amplitude of the oscillation increases with increasing concentration, while the length scale of the oscillation decreases, in agreement with experiment. ${ }^{19}$

The strong attraction at short distances is due to depletion of both the polyelectrolyte and the counterions. To demonstrate this, in Fig. 4, we show the concentration profiles of the polymer scaled by its bulk concentration near a single plate. As expected, the polymer is depleted in the vicinity of the surface due to conformation entropy penalty. The small counterions are also depleted near the surface, but to a considerably less degree. The difference in the polymer and counterion profiles leads to local charge separation, with more positive charge next to the surface. This local charge separation gives rise to an effective electrical double layer and is also responsible for the appearance of an "adsorptionlike" feature next to the depletion region, with density higher than the bulk density. We observe that the range of the polymer depletion layer decreases with increasing polymer concentration due to the increased osmotic pressure of the polyelectrolytes.

When two surfaces approach each other to a distance on the order of the depletion layer thickness, two competing effects are at play. On the one hand, the overlap of the double layers on the two surfaces gives rise to a double-layer repulsion. This can be seen from the increase in the electrostatic potential with decreasing separation; see Fig. 5. On the other hand, the overall polymer density (and the counterion density, due to charge neutrality within the slits) decreases, resulting in a depletion attraction. Figure 6 shows

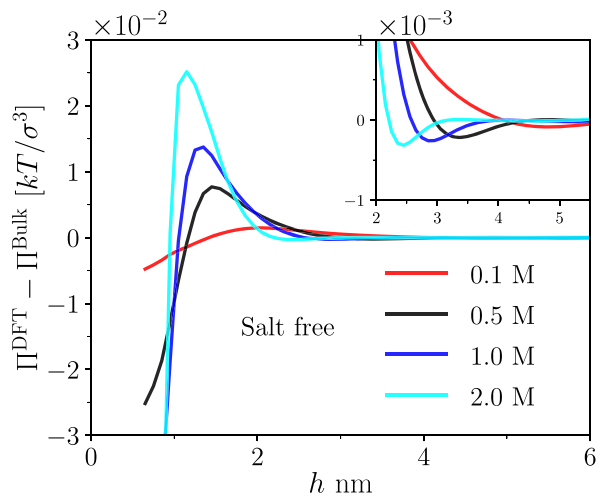

FIG. 3. Polyelectrolyte-mediated interactions between two neutral hard wall surfaces corresponding to different monomer concentrations. The dielectric constant of the system is uniform and fixed on $\epsilon=80$; the temperature is $300 \mathrm{~K}$; the polyelectrolyte consists of 60 -mers, and the diameter of the monomers and counterions is $\sigma=5 \AA$. The inset displays the long-range attractions.
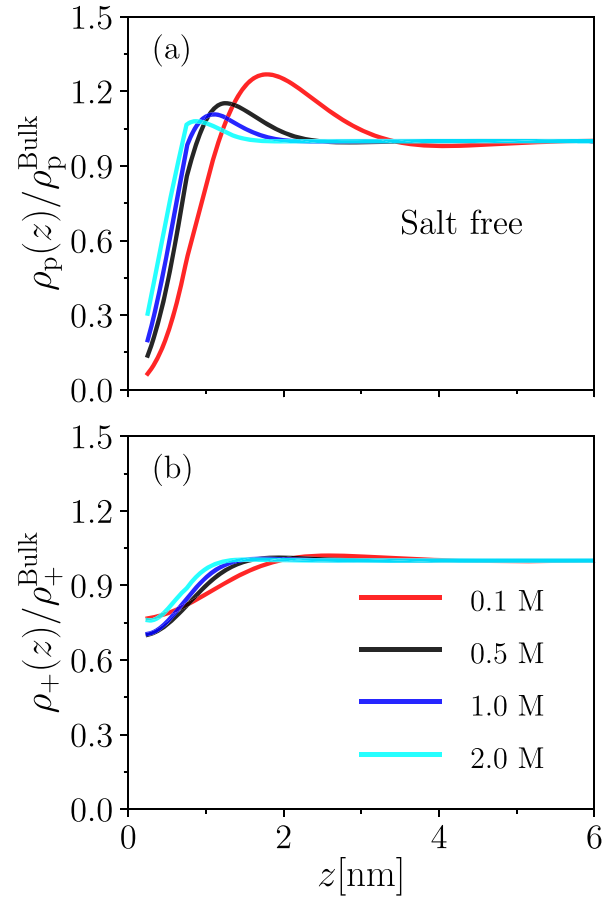

FIG. 4. Density profiles for monomers (a) and counterions (b) of the 60-mers polyelectrolytes for a salt-free system in a slit pore (neutral hard wall surfaces) with uniform dielectric constant $\epsilon=80$. The diameter of the monomers and counterions is $\sigma=5 \AA$, and the temperature is $300 \mathrm{~K}$. The legends indicate the bulk monomer concentrations.

the overall density $\bar{N}(h)$ of the polymers in the slit as a function of the separation $h$. With moderate depletion, the interaction is still dominated by the double-layer repulsion. However, at sufficiently small distances, the density in the gap becomes sufficiently low that depletion attraction dominates. The competition between these two effects results in the nonmonotonic behavior in the force profile. We remind the reader that the nature of the interaction between two neutral surfaces in the presence of polyelectrolyte solutions has been

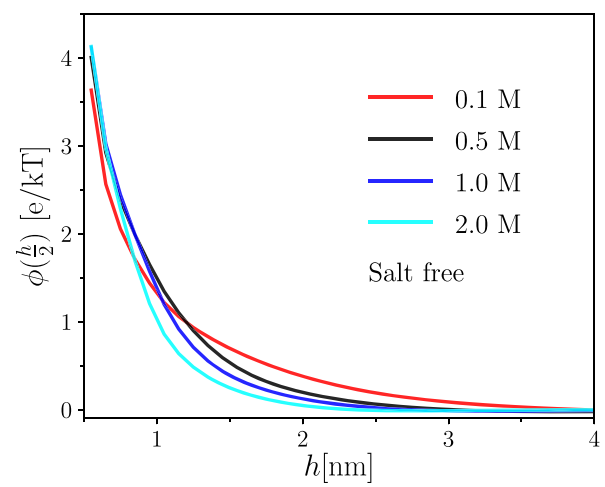

FIG. 5. The electric potentials in the central region of the slit pores. All of the parameters are the same with those of Fig. 4. 


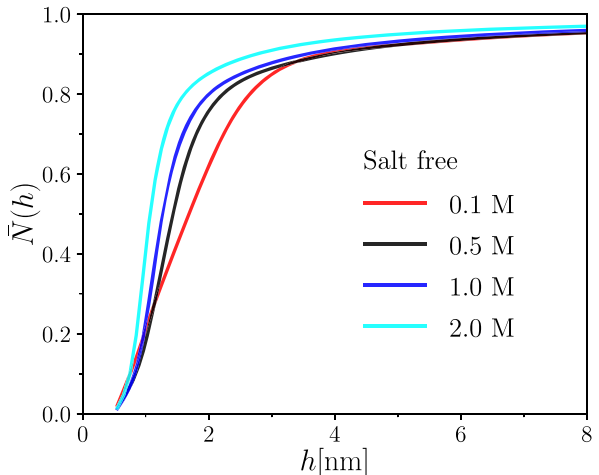

FIG. 6. The overall density profiles $\bar{N}(h)$ for monomers in slit pores with different sizes, where $\bar{N}(h)=\int_{0}^{h} \mathrm{~d} z \rho_{\mathrm{p}}(z) / \rho_{\mathrm{p}}^{\text {Bulk }}\left(h-\sigma_{\mathrm{p}}\right)$. All of the parameters are the same with those of Fig. 4.

quite controversial in the literature. Yethiraj, using integral equation theory, predicted an oscillatory profile with strong repulsion at short distances. ${ }^{21}$ On the one hand, using both Monte Carlo simulation and density functional theory, Jönsson and co-workers similarly reported strong short-range repulsion followed by attraction. ${ }^{11}$ On the other hand, a different study by Turesson et al. using MC simulations at constant surface charge density ${ }^{26}$ found that when the surface charge density is relatively low (say, $1 \mathrm{e}_{0} / \mathrm{nm}^{2}$ ), the force profile has short-range attraction followed by repulsion. Pryamitsyn and Ganesan also found that when the surfaces are neutral or weakly charged, the polymer-mediated interactions predominantly consist of a short-range attraction followed by long-range repulsion. ${ }^{30}$ Our calculations support the latter two studies.

The key features of the interaction force profile for the salt-free systems can be captured without accounting for electrostatic correlations. For comparison, we have performed a calculation where the electrostatic interactions are treated at the Poisson-Boltzmann (PB) mean-field level. In Fig. 7, we compare the density and force (inset) profiles calculated, respectively, from our full DFT and from PB. We see that the qualitative shapes of these two set of curves are quite

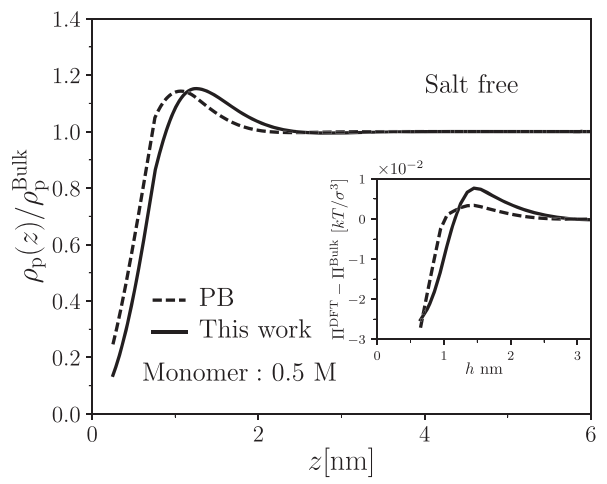

FIG. 7. Density profiles for monomers predicted from our full PDFT and PB. The parameters are the same as the ones in Fig. 4. The inset displays the interactions for the case when the monomer concentration is $0.5 \mathrm{M}$. similar; this is because both the charge separation and the depletion are captured at the PB level. However, both the depletion and the "adsorptionlike" peak in the density profiles are made more pronounced due to electrostatic correlations; these correlations enhance the attraction between the polymers and the counterions, and also contribute to more depletion near the surface even for small ions. Furthermore, the faint secondary attractive well shown in the inset of Fig. 4 is absent in the PB treatment. This well becomes much more pronounced when there is salt in the solution, and as we demonstrate in Sec. IV B, it is a manifestation of the electrostatic correlations.

This conclusion indicates that for salt free systems, mean field level electric-double-layer (EDL) dominates the properties. However, for the systems with salt, the results from PB theory can be qualitatively different from the results from our theory.

\section{B. Systems with salt}

In this section, we discuss the interaction of polyelectrolyte solutions with added salts. We start with the effect of chain lengths. In Fig. 8, we present the interaction force for a polyelectrolyte solution at $0.5 \mathrm{M}$ (monomer concentration) polyelectrolytes and $3 \mathrm{M}$ salts for several different chain lengths. Compared to Fig. 2(b), there is more variation for the shorter chain lengths $(N=10$ and $N=20)$, but the curves for $N=60$ and $N=100$ are nearly the same. So, even for this relatively higher salt concentration, the chain length dependence becomes rather weak once the chains are moderately long. In the rest of our discussions, we will focus on the $N=60$ system.

In Fig. 9, we show the force profiles in a $0.5 \mathrm{M}$ polyelectrolyte solution with varying salt concentrations. In all cases, the force exhibits strong short-range attraction followed by a repulsive peak, which agrees with previous studies. ${ }^{26,30}$ With increasing salt concentration, the location of the repulsive peak is seen to shift to smaller separation due to the reduction in screening length with increasing salt concentration. However, the peak height changes nonmonotonically-first decreasing and then increasingwith increasing salt concentration due to the complicated competition between the EDL overlapping and the electrostatic

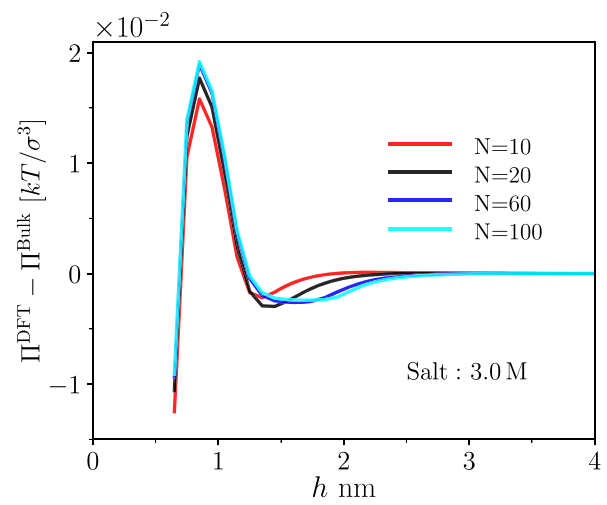

FIG. 8. Polyelectrolyte-mediated interactions between two neutral hard wall surfaces corresponding to different chain lengths. The dielectric constant of the system is uniform and fixed on $\epsilon=80$; the temperature is $300 \mathrm{~K}$; the polymer and salt concentrations are $0.5 \mathrm{M}$ and $3 \mathrm{M}$, respectively; the diameter of the monomers and counterions is $\sigma=5 \AA$. 


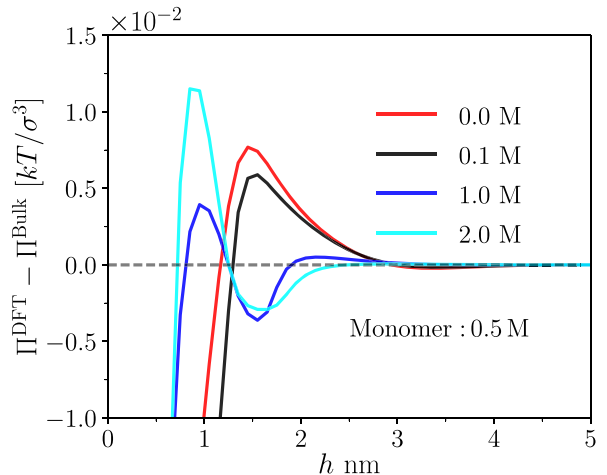

FIG. 9. Polyelectrolyte-mediated interactions corresponding to different salt concentrations. The monomer concentration is fixed on $0.5 \mathrm{M}$. The other parameters are the same with the ones in Fig. 8.

correlations; this will be elaborated later. The faint secondary attractive well observed for the salt-free case (the red curve in Fig. 9) is now much more pronounced due to the electrostatic correlations, and the well depth varies nonmonotonically with concentration: The well depth first increases and then decreases.

The strong attraction at short distances is clearly due to depletion. Figure 10 shows the monomer concentration profile near a single surface. Interestingly, the range of polymer depletion increases with salt concentration (but eventually approaches the neutral polymer limit for very high salt concentration). That there is less depletion for polyelectrolyte at lower salt concentrations is due to the attraction between the negatively charged polymer and the positive charges in the region next to the surface. The degree of charge separation decreases with increasing salt concentration due to screening, eventually leading to the disappearance of the "adsorptionlike" feature seen for the salt-free and low salt concentrations (e.g., 0.1M).

Similar to the salt-free case, the presence of local charge separation near the surface generates an effective double layer, which is partially responsible for the repulsive peak in the force profile.

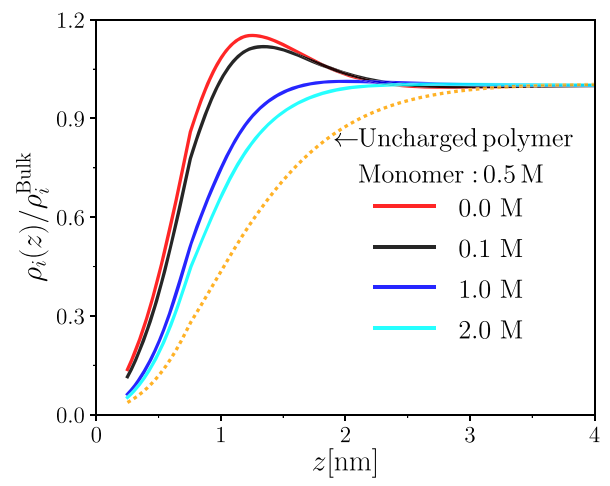

FIG. 10. The density profiles for the monomers in the conditions of different salt concentrations near a single neutral surface. The parameters are the same as the ones in Fig. 8.

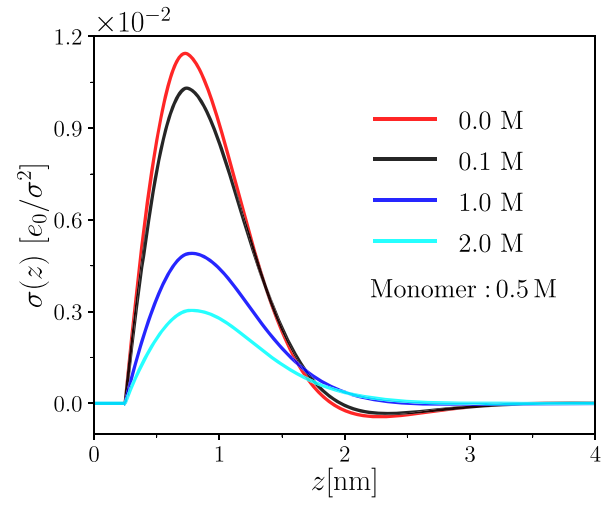

FIG. 11. The net charge distribution in the conditions of different salt concentrations near a single neutral surface. The parameters are the same with the ones in Fig. 8.

To quantify the degree of charge separation, we define the net accumulated charge per unit area at a distance $z$ from the surface by

$$
\sigma(z)=\sum_{i} \int_{0^{+}}^{z} Z_{i} e_{0} \rho_{i}\left(z^{\prime}\right) \mathrm{d} z^{\prime} .
$$

The result is shown in Fig. 11 for several salt concentrations. In all cases, the net charge starts from zero at the surface and increases positively, reflecting the excess (relative to the polyelectrolyte) of the positively charged counterions in the region near the surface. At larger distances, the excess positive charge is compensated by the negatively polyelectrolytes, and the neutralization becomes complete sufficiently far away from the surface. It is interesting that for salt-free and low salt concentration, $\sigma$ actually turns negative before reaching 0 , while for moderate and high salt concentrations, $\sigma$ approaches 0 from above. The appearance of the negative $\sigma$ is correlated with the appearance of the "adsorptionlike" peak in the polymer density profile. On the one hand, as can be seen from Fig. 11, with increasing salt concentration, the peak value of $\sigma$ decreases, signaling less charge separation. On the other hand, from Fig. 9, we see that the repulsive peak increases with increasing salt concentration (at higher salt concentrations). Thus, while the charge-separation induced double layer should contribute partially to the repulsive peak in the force, it does not explain the trend with the salt concentration. On the contrary, electrostatic correlations become stronger with higher salt concentrations, and the stronger electrostatic correlations will enhance the repulsion barrier. Therefore, the competition between the EDL and the electrostatic correlations results in the nonmonotonic behavior. When the salt concentration is low (roughly less than 1.0M), the EDL dominates the interaction potentials. Hence, the repulsion barrier will become weaker with increasing salt concentrations in the EDL-dominant regime. When the salt concentration is high (roughly more than $1.0 \mathrm{M})$, the interaction becomes dominated by electrostatic correlations. The repulsion barrier increases with increasing salt concentrations.

The interaction between the two surfaces in the case of $1 \mathrm{M}$ salt concentration shows prominent oscillation with the appearance of a second repulsion peak; see Fig. 9. In order to understand the origin 


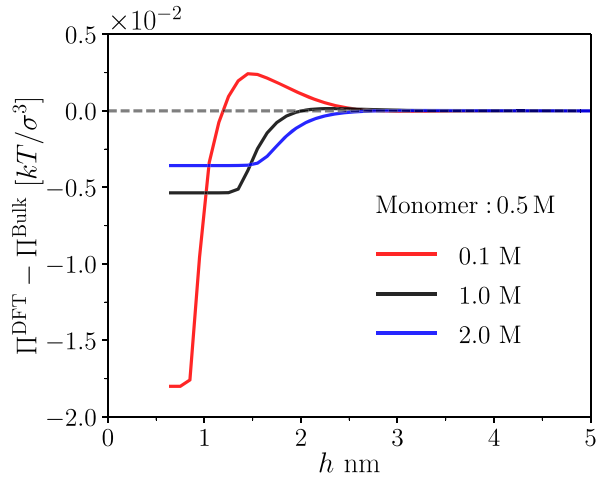

FIG. 12. Polyelectrolyte-mediated interactions predicted by PB equation. The monomer concentration are fixed on $0.5 \mathrm{M}$. The other parameters are the same with the ones in Fig. 8.

of this oscillatory interaction, we compare our results with a corresponding calculation from the mean-field $\mathrm{PB}$ theory, which does not include any electrostatic correlations. As seen in Fig. 12, the PB theory predicts neither the second attractive well nor the second repulsion barrier. The strength of the repulsion barrier decreases with increasing salt concentrations; in contrast to our PDFT predictions shown in Fig. 9. When the salt concentration increases to $2 \mathrm{M}$, the $\mathrm{PB}$ theory predicts pure depletion-attraction, which is similar to the one induced by uncharged polymer solutions; see Fig. 2(a). Since the only difference between the PB theory and the PDFT is that the PB theory does not include electrostatic correlations, we conclude that the missing electrostatic correlations (from both the small mobile ions and the chain connectivities) in the PB theory plays a key role in determining the interaction behaviors between two surfaces mediated by polyelectrolyte solutions at moderate to high salt concentrations.

\section{CONCLUSION}

In this work, we propose a polymer density functional theory to study the interactions between two planar surfaces in polyelectrolyte solutions. To focus on the origin of the complex interaction behaviors, we consider structureless hard walls and treat the solvent using an incompressibility condition within a continuum dielectric background.

In the absence of salts, our results indicate that the interaction primarily consists of a depletionlike attraction at short distance followed by a repulsion barrier at longer distance. A second attractive well is also observed in the cases with high polyelectrolyte concentrations. Furthermore, with increasing the polyelectrolyte concentration, both the strength of the attractive well and the repulsive peak are also increased. These results are consistent with the experimental observations, ${ }^{19}$ simulation results, ${ }^{26}$ and theoretical calculations. ${ }^{30}$

For the cases with salts, our results show that the interactions are determined by the overlap between electrical double layers from each surface as a result of charge separation when the salt concentration is low. However, for the systems with higher salt concentrations, the interaction behaviors are affected by the electrostatic correlations. The competition between the strength of the electric double layer and the electrostatic correlations makes the magnitude of the repulsion barrier vary nonmonotonically, while the self-consistent field theory predicts the monotonically decreasing repulsion barrier. For intermediate salt concentrations, oscillatory interactions are observed, and by comparison with the mean-field PB treatment of electrostatics, we conclude that the origin of the oscillation is the electrostatic correlations.

We should comment that, although our theoretical calculations capture the qualitative force behaviors observed in experiments and simulations, significant quantitative discrepancies exist, especially with the experiment results. For instance, the interaction range and the period of the oscillation predicted by our work $(\sim 3 \mathrm{~nm})$ are much smaller than the lengths observed in experiments (usually $10 \mathrm{~s}$ of $\mathrm{nm}$ ). Aside from the fact that experiments were usually performed at much lower concentrations, these discrepancies reflect the limitation of our DFT. As we have alluded to in the presentation of the theory, the DFT does not correctly capture the chain conformation at dilute and semidilute conditions and underestimates the electrostatic correlation effects. In particular, by neglecting the local rigidity on the blob size scale, the DFT would greatly underestimate the correlation length of the solution, which has been shown to be the length scales of the interactions. $4,5,13,21-24,32,60$ Nevertheless, insofar as the essential physics is concerned, we believe many of the qualitative findings drawn from this approximate theory at high concentrations are relevant to the experiments and simulations at lower concentrations.

In summary, the interaction between hard walls mediated by polyelectrolyte solutions results from a complex interplay among three factors: the depletion of polymers near the surface, electrical double layer due to charge separation near the surface, and electrostatic correlations. While the first two factors can be captured with mean-field theories, such as self-consistent field theory, electrostatic correlations can qualitatively alter the force behavior. The PDFT proposed in this work accounts for all three factors (though approximately) and thus allows us to understand the observed behavior in the interaction forces. In this work, we have focused on the simplest surfaces, namely, hard walls with no interactions with any species. In future work, we will explore how electrostatic and nonelectrostatic interactions between the polymer and the surface modulate the effective interactions between the surfaces.

\section{ACKNOWLEDGMENTS}

The Dow Chemical Company is acknowledged for funding and for permission to publish the results.

\section{REFERENCES}

${ }^{1}$ T. Åkesson, C. E. Woodward, and B. Jönsson, J. Chem. Phys. 91, 2461-2469 (1989).

${ }^{2}$ S. J. Miklavic, C. E. Woodward, B. Jönsson, and T. Åkesson, Macromolecules 23, 4149-4157 (1990).

${ }^{3}$ R. von Klitzing, Adv. Colloid Interface Sci. 114-115, 253-266 (2005).

${ }^{4}$ C. Üzüm, S. Christau, and R. von Klitzing, Macromolecules 44, 7782-7791 (2011).

${ }^{5}$ C. Üzüm, R. Makuska, and R. von Klitzing, Macromolecules 45, 3168-3176 (2012).

${ }^{6}$ M. Borkovec, I. Szilagyi, I. Popa, M. Finessi, P. Sinha, P. Maroni, and G. Papastavrou, Adv. Colloid Interface Sci. 179-182, 85-98 (2012). 
${ }^{7}$ B. Bolto and J. Gregory, Water Res. 41, 2301-2324 (2007).

${ }^{8}$ A. M. Howe, R. D. Wesley, M. Bertrand, M. Co̊te, and J. Leroy, Langmuir 22, 4518-4525 (2006).

${ }^{9}$ P. M. Claesson, A. Dedinaite, and O. J. Rojas, Adv. Colloid Interface Sci. 104, 53-74 (2003).

${ }^{10}$ A. J. Milling and K. Kendall, Langmuir 16, 5106-5115 (2000).

${ }^{11}$ B. Jönsson, A. Broukhno, J. Forsman, and T. Åkesson, Langmuir 19, 9914-9922 (2003).

${ }^{12}$ O. Théodoly, J. S. Tan, R. Ober, C. E. Williams, and V. Bergeron, Langmuir 17, 4910-4918 (2001)

${ }^{13}$ D. Qu, J. S. Pedersen, S. Garnier, A. Laschewsky, H. Möhwald, and R. von Klitzing, Macromolecules 39, 7364-7371 (2006).

${ }^{14}$ I. K. Snook and W. van Megen, J. Chem. Phys. 72, 2907-2913 (1980).

${ }^{15}$ D. Henderson and M. Lozada-Cassou, J. Colloid Interface Sci. 114, 180-183 (1986).

${ }^{16}$ H. K. Christenson, D. W. R. Gruen, R. G. Horn, and J. N. Israelachvili, J. Chem. Phys. 87, 1834-1841 (1987).

${ }^{17}$ Intermolecular and Surface Forces, edited by J. N. Israelachvili (Academic Press, Boston, 2011)

${ }^{18}$ V. Bergeron, D. Langevin, and A. Asnacios, Langmuir 12, 1550-1556 (1996).

${ }^{19}$ A. J. Milling, J. Phys. Chem. 100, 8986-8993 (1996).

${ }^{20}$ A. Asnacios, A. Espert, A. Colin, and D. Langevin, Phys. Rev. Lett. 78, 49744977 (1997).

${ }^{21}$ A. Yethiraj, J. Chem. Phys. 111, 1797-1800 (1999).

${ }^{22}$ R. von Klitzing, A. Espert, A. Asnacios, T. Hellweg, A. Colin, and D. Langevin, Colloids Surf. A 149, 131-140 (1999).

${ }^{23}$ B. Kolarić, W. Jaeger, and R. von Klitzing, J. Phys. Chem. B 104, 5096-5101 (2000).

${ }^{24}$ R. von Klitzing, B. Kolarić, W. Jaeger, and A. Brandt, Phys. Chem. Chem. Phys. 4, 1907-1914 (2002).

${ }^{25}$ A. V. Dobrynin and M. Rubinstein, Prog. Polym. Sci. 30, 1049-1118 (2005).

${ }^{26} \mathrm{M}$. Turesson, T. Åkesson, and J. Forsman, Langmuir 23, 9555-9558 (2007).

${ }^{27}$ S. H. L. Klapp, D. Qu, and R. von Klitzing, J. Phys. Chem. B 111, 1296-1303 (2007).

${ }^{28}$ M. Turesson, C. E. Woodward, T. Åkesson, and J. Forsman, J. Phys. Chem. B 112, 5116-5125 (2008).

${ }^{29}$ D. Qu, D. Baigl, C. E. Williams, H. Möhwald, and A. Fery, Macromolecules 36, 6878-6883 (2003)

${ }^{30}$ V. Pryamitsyn and V. Ganesan, Macromolecules 47, 6095-6112 (2014).

${ }^{31}$ M. A. Carignano and N. Dan, Langmuir 14, 3475-3478 (1998).

${ }^{32}$ C. Üzüm, N. Kristen, and R. von Klitzing, Curr. Opin. Colloid Interface Sci. 15, 303 (2010).

${ }^{33}$ J. Jiang, V. V. Ginzburg, and Z.-G. Wang, Soft Matter 14, 5878-5887 (2018).

${ }^{34}$ C. E. Woodward, J. Chem. Phys. 94, 3183-3191 (1991).

${ }^{35} \mathrm{Z}$. Li and J. Wu, Phys. Rev. Lett. 96, 048302-048305 (2006).

${ }^{36} \mathrm{~T}$. Jiang, Z. Li, and J. Wu, Macromolecules 40, 334-343 (2007).

${ }^{37}$ G. Fredrickson, The Equilibrium Theory of Inhomogeneous Polymers (Oxford University Press, USA, 2006).

${ }^{38}$ J. Forsman, C. E. Woodward, and B. C. Freasier, J. Chem. Phys. 118, 7672-7681 (2003).

${ }^{39}$ J. Forsman and C. E. Woodward, Macromolecules 39, 1269-1278 (2006).
${ }^{40}$ J. Forsman and C. E. Woodward, Macromolecules 40, 8396-8408 (2007).

${ }^{41}$ C. N. Patra and A. Yethiraj, J. Chem. Phys. 112, 1579-1584 (2000).

${ }^{42}$ L. Blum, Mol. Phys. 30, 1529-1535 (1975).

${ }^{43}$ L. Blum and J. S. Hoeye, J. Phys. Chem. 81, 1311-1316 (1977).

${ }^{44}$ K. Hiroike, Mol. Phys. 33, 1195-1198 (1977).

${ }^{45}$ L. Blum and Y. Rosenfeld, J. Stat. Phys. 63, 1177-1190 (1991).

${ }^{46}$ Y.-X. Yu, J. Wu, and G.-H. Gao, J. Chem. Phys. 120, 7223-7233 (2004).

${ }^{47}$ D. Gillespie, M. Valiskó, and D. Boda, J. Phys.: Condens. Matter 17, 6609-6626 (2005).

${ }^{48}$ J. Jiang, D. Cao, D. Henderson, and J. Wu, J. Chem. Phys. 140, 044714-044722 (2014).

${ }^{49}$ D. Gillespie, W. Nonner, and R. S. Eisenberg, J. Phys.: Condens. Matter 14, 12129-12145 (2002).

${ }^{50}$ M. S. Wertheim, J. Stat. Phys. 35, 19-34 (1984).

${ }^{51}$ M. S. Wertheim, J. Stat. Phys. 35, 35-47 (1984).

${ }^{52}$ M. S. Wertheim, J. Stat. Phys. 42, 459-476 (1986).

${ }^{53}$ M. S. Wertheim, J. Chem. Phys. 85, 2929-2936 (1986).

${ }^{54}$ M. S. Wertheim, J. Chem. Phys. 88, 1145-1155 (1988).

${ }^{55}$ E. Kierlik and M. L. Rosinberg, J. Chem. Phys. 100, 1716-1730 (1994).

${ }^{56}$ C. J. Segura, W. G. Chapman, and K. P. Shukla, Mol. Phys. 90, 759-772 (1997).

${ }^{57}$ C. J. Segura, E. V. Vakarin, W. G. Chapman, and M. F. Holovko, J. Chem. Phys. 108, 4837-4848 (1998).

${ }^{58}$ Y.-X. Yu and J. Wu, J. Chem. Phys. 116, 7094-7103 (2002).

${ }^{59}$ Y.-X. Yu and J. Wu, J. Chem. Phys. 117, 2368-2376 (2002).

${ }^{60}$ A. V. Dobrynin, R. H. Colby, and M. Rubinstein, Macromolecules 28, 18591871 (1995).

${ }^{61}$ M. Muthukumar, J. Chem. Phys. 105, 5183-5199 (1996).

${ }^{62}$ K. Shen and Z.-G. Wang, J. Chem. Phys. 146, 084901-084916 (2017).

${ }^{63}$ P. Tarazona, Mol. Phys. 52, 81-96 (1984).

${ }^{64}$ P. Tarazona and R. Evans, Mol. Phys. 52, 847-857 (1984).

${ }^{65}$ P. Tarazona, Phys. Rev. A 31, 2672-2679 (1985).

${ }^{66}$ A. R. Denton and N. W. Ashcroft, Phys. Rev. A 39, 4701-4708 (1989).

${ }^{67}$ C. N. Patra and S. K. Ghosh, J. Chem. Phys. 116, 8509-8516 (2002).

${ }^{68}$ A. R. Denton and N. W. Ashcroft, Phys. Rev. A 42, 7312-7329 (1990).

${ }^{69}$ R. L. Davidchack and B. B. Laird, Phys. Rev. E 60, 3417-3420 (1999).

${ }^{70} \mathrm{R}$. Leidl and H. Wagner, J. Chem. Phys. 98, 4142-4148 (1993).

${ }^{71}$ Y. Rosenfeld, Phys. Rev. Lett. 63, 980-983 (1989).

${ }^{72}$ P. Tarazona, Phys. Rev. Lett. 84, 694-697 (2000).

${ }^{73}$ Y.-X. Yu and J. Wu, J. Chem. Phys. 117, 10156-10164 (2002).

${ }^{74}$ R. Roth, R. Evans, A. Lang, and G. Kahl, J. Phys.: Condens. Matter 14, 12063 12078 (2002).

${ }^{75} \mathrm{P}$. Tarazona and R. Evans, Mol. Phys. 47, 1033-1063 (1982).

${ }^{76}$ P. Tarazona, J. A. Cuesta, and Y. Martínez-Ratón, Long Ranged Correlations at a Solid-Fluid Interface A Signature of the Approach to Complete Wetting (Springer, Berlin, Heidelberg, 2008).

${ }^{77}$ G. A. Mansoori, N. F. Carnahan, K. E. Starling, and T. W. Leland, J. Chem, Phys. 54, 1523-1525 (1971).

${ }^{78}$ T. Boublík, J. Chem. Phys. 53, 471-472 (1970).

${ }^{79}$ J. F. Joanny, L. Leibler, and P. G. De Gennes, J. Polym. Sci. Part B: Polym. Phys. 17, 1073-1084 (1979).

${ }^{80}$ S. Asakura and F. Oosawa, J. Chem. Phys. 22, 1255-1256 (1954). 